\title{
Carmen Perujo, una historia de vida (I). Nacimiento en la preguerra y años de formación de una mujer artista plástica en la postguerra española (1930-1951)
}

\author{
Pilar Vicente de Foronda ${ }^{1}$
}

Resumen. Este artículo presenta la biografía contextualizada de Carmen Perujo Guerrero, escultora española nacida en 1930, en Sevilla, desde el momento de su nacimiento hasta que termina sus estudios en la escuela Santa Isabel de Hungría en Sevilla. Se quedan, para posteriores artículos, los años de madurez artística y personal en que triunfa como ceramista escultora tanto económicamente como a nivel de la crítica artística recibida. Se analiza desde los gender studies y desde la metodología de la historia de vida, incluyendo las circunstancias sociopolíticas que la rodearon desde el momento de su nacimiento, estudios y primera juventud. Dada la escasez de genealogía de mujeres artistas que crearon su obra durante el periodo de la dictadura y la transición a la democracia, este artículo viene a poner en valor esta artista excepcional que, habiendo recibido los ecos de las mujeres del primer tercio de siglo, supo sobreponerse al papel de ángel del hogar para ella adjudicado por el régimen y crear una obra personal que ha servido de referencia para la generación siguiente.

Palabras clave: estudios de género; invisibilidad; mujeres artistas; escultoras

\section{[en] Carmen Perujo, a history of life Birth in the pre-war period and formation years of a plastic artista woman in the Spanish post-war period $(1930-1951)$}

\begin{abstract}
This article presents the contextualized biography of Carmen Perujo Guerrero, Spanish sculptor born in 1930, in Seville, from the moment of her birth until the end of artistic formation in the Santa Isabel de Hungría school in Sevilla. Left, for later articles, the years of personal maturity, in which she succeeds as a ceramic sculptor both economically and in the artist critics. It is analyzed from the gender studies perspective and from the life history methodology, including the sociopolitical circumstances that surrounded her from the moment of her birth, studies and first youth. Given the lack of female artists' genealogy who created her work during the period of the dictatorship and the transition to democracy, this article enhances the work of this exceptional artist that received the echoes of the women of the first third of the century and knew how to overcome the role of household angel that was awarded to her, as for all women, by the regime and created a personal work that has served as a reference for the next generation.
\end{abstract}

Keywords: gender studies; invisibility; woman artists; sculptress.

Sumario: 1. Introducción. 2. Nacimiento en Sevilla. Infancia y Guerra Civil. 3. Juventud y estudios. 4. Conclusiones. Referencias bibliográficas.

\footnotetext{
$1 \quad$ Universidad Complutense de Madrid

Universidad de Granada

info@foronda.es
} 
Cómo citar: Vicente de Foronda. P. (2018). Carmen Perujo, una historia de vida (I). Nacimiento en la preguerra y años de formación de una mujer artista plástica en la postguerra española (1930-1951), Investigaciones feministas 9.1, 83-100

El punto de vista sobre el pasado, la manipulación de la memoria por todos aquellos que se consagran sucesivamente a narrar el pasado, nunca es inocente (DUBY, G. 1994:78)

\section{Introducción}

El objeto de este artículo es visibilizar la vida de la escultora ceramista Carmen Perujo como ejemplo paradigmático de la supervivencia de las mujeres artistas en la España del primer franquismo. Su visibilización como ceramista escultora y la descripción y el estudio detallado de la vida y la obra de una mujer artista que vive la Guerra Civil en su primera infancia y, habiendo nacido ciudadana, es súbdita durante la madurez de su vida para llegar al éxito profesional, pudiendo realizar su vocación artística en plenitud. Excluimos del relato sus años de éxito y mayor producción, dado que tienen lugar en los años 70, periodo alejado de la postguerra. Este artículo se centra en sus años de infancia, formación y de las circunstancias sociopolíticas que la rodearon y la conformaron como artista.

Narrando la historia de vida de Carmen Perujo y las circunstancias sociopolíticas que la rodeaban se puede comprender mejor la historia vital de las mujeres artistas de la siguiente generación, nacidas en la segunda mitad del siglo XX. Poner en valor el legado escultórico de Carmen Perujo es un acto de justicia democrática necesaria para comprender como, a pesar del borrado ideológico y de la supresión de la libertad de pensamiento, hubo lugares de resistencia para la creatividad que son los que permitieron que el fino hilo de la genealogía de las mujeres no quedara definitivamente roto.

Los cortes de genealogía son un daño que las sociedades tardan generaciones en curar y que en la genealogía de las mujeres sucede con demasiada frecuencia. El secuestro genealógico, que siglo tras siglo se comete con las mujeres, les impide la posibilidad de tener la capacidad simbólica para ser sujetos de creación, evitando ser objeto de creación. Los estudios de género encargados de recuperar la genealogía de creación y de pensamiento de las mujeres, tienen cincuenta años de existencia y es a estos gender studies que este artículo se acoge. También intenta aportar algo de luz sobre los estudios de género en la España de la postguerra, donde todavía es muy necesario dar luz al periodo posterior a la Guerra Civil hasta los tiempos de la transición.

\section{Nacimiento en Sevilla. Infancia y Guerra Civil}

Carmen Perujo ${ }^{2}$ nace el 7 de marzo de 1930, en Sevilla. Es bautizada en Santa María la Blanca como Ana de la Paz Carmen Perujo Guerrero. Su padre, Esteban Perujo

La documentación personal de Carmen Perujo se encuentra en los anexos de la tesis Carmen Perujo, una historia de vida. Catálogo razonado de su obra escultórica. 40 escultoras españolas del siglo XX, en proceso de publicación. 
Ortega, jornalero del campo que emigra a la Argentina de joven y vuelve posteriormente a su pueblo, Cuevas del Becerro, para casarse con Presentación Guerrero, nacida en el mismo pueblo ubicado en la Serranía de Ronda para posteriormente trasladarse a vivir a Sevilla. Aunque Carmen nace en la calle de Las Cruces, su familia pronto se trasladará a la calle del Aire, 18, donde su padre y su madre abren una pensión, como cuenta Carmen, justo frente al que, en aquel momento, era el domicilio del poeta Luís Cernuda. Carmen es del Barrio de Santa Cruz: de la calle Aire, en una casa con patio andaluz de dos plantas, la Pensión Santa Cruz, nombre de la pequeña empresa familiar en la que trabajan sus padres y sus hermanas mayores, Pastora, Rosa y Ángeles. Esteban y Presentación se dedican a la crianza de sus cuatro hijas, siendo Carmen la más pequeña.

Carmen Perujo nace el mismo año que Amalia Avia, Julio López Hernández y Antonio Saura. El mismo año que Carmen Jiménez, escultora sevillana, se queda huérfana y pasa, en primer lugar, a vivir con sus tíos para terminar interna en el Colegio de huérfanos, General Riquelme de Granada ${ }^{3}$. Nace en un momento de grandes cambios tanto a nivel mundial como nacional, viene al mundo justo cuando las mujeres españolas se hacen ciudadanas para pasar a ser súbdita con 6 años y recuperar la ciudadanía a los 58 años.

Carmen tiene una salud delicada de pequeña y pasa largas temporadas en el campo. De los cuatro a los veinte años, vive de dos a tres meses en las huertas de Montellano, como un miembro más, con una familia de humildes labradores, amiga de los padres. Sus citas con el campo de Montellano no se interrumpen en toda la infancia y primera juventud, formando su carácter y desarrollando su pasión de contacto directo con la naturaleza y con los problemas del campo andaluz y sus gentes. Carmen tiene escrito: quien haya vivido allí, sabrá del surco de las palmas, entre el limpio colorido de las macetas, el tañer de las campanas y el aleteo de los vencejos.

Los cambios que están sucediendo en este primer tercio de siglo en el que nace Carmen Perujo son de enorme trascendencia para las mujeres que, además de lograr la ciudadanía en algunos países, van accediendo a la cultura, siendo así que las primeras licenciadas y profesionales van desarrollando por esos años sus vidas profesionales. Valgan como muestra la Asociación Universitaria Femenina, fundada en 1920, y el Lyceum Club Femenino, de 1926, o la Residencia de Señoritas que abre paso a la educación superior para las mujeres ${ }^{4}$ aunque solo fuera para unas pocas, era un buen principio que se vió truncado por el golpe de estado de 1936.

La ciudadanía, que en algunos momentos, se entendió como un cambio definitivamente consolidado, es algo excepcional en la historia y todavía se halla en cuestión en muchos lugares del mundo. Los modos de vida de las mujeres españolas habían experimentado grandes cambios en torno a 1920. Las mujeres aprovechan las oportunidades que se les daban para salir de lo privado a lo público: a los salones de té, los clubes, los grandes hoteles, donde pueden fumar y vestir a la moda, aun cuando no conocen de las libertades sociales o educativas de las mujeres en el resto de Europa. La neutralidad de España en la Gran Guerra tuvo como consecuencia que no sustituyéramos a los hombres en el trabajo. La opinión liberal instruida se había opuesto cada vez más, desde la década de 1920, a la idea tradicional de las mujeres

Pareja y Márquez, 1994.

4 Richmond, 2004, 34 
expuesta por la Iglesia católica, principal suministradora de educación secundaria 5 . Perujo es deudora de estos cambios que, incluso 20 años después de haber tenido lugar y haber desaparecido, todavía tenían ecos: se formará como pintora en la Facultad de Bellas Artes de Sevilla en los años de la postguerra, para concluir siendo ceramista escultora en Majadahonda, provincia de Madrid.

A principios del siglo XX nuestro país tiene una población de 18.618.086 habitantes. En el año de nacimiento de Carmen Perujo, esta cifra se habrá incrementado a 23.677974, como explica Rosa Capel en El trabajo y la educación de la mujer en España. En 1930 España seguía siendo en gran parte rural y semiurbana y el modelo de vida de la mayoría de mujeres del campo apenas había cambiado. El empleo femenino, fundamentalmente agrícola, en el servicio doméstico y en las fábricas, en zonas urbanas, suponía en 1930 el 12, $6 \%$ de la mano de obra nacional. En este mismo año el analfabetismo en España es de un 42,2 \%. Entre la población femenina se reparte casi al $50 \%$, aunque prevalecen las que saben leer y escribir. Eran 6.070.609, el 50,1\%, más el 1,1 que solo sabía leer, frente a 5.754.569, el 47,5 por ciento, totalmente analfabetas ${ }^{6}$.

En el caso concreto de Sevilla, en 1930, un 51,4\% de las mujeres no sabe leer ni escribir". En 1930 la educación de las mujeres alcanza el 13,3 \% de los "bachilleres" y casi la cuarta parte de los matriculados en las enseñanzas profesionales ${ }^{8}$. El Bachillerato, cursado a lo largo de seis años académicos tal como decía la Ley de 1901, era o la antesala de la Universidad o el modo de lograr un empleo con algo de cualificación o bien servía para satisfacer deseos de cultivo intelectual en el caso de las clases más pudientes.

En Sevilla, lugar de nacimiento de Carmen, es la Sociedad Económica de Amigos del País, creada en el siglo XVIII, la que supone la reunificación de los ciudadanos con interés por las ciencias, las artes y la industria. Esta Sociedad, a pesar de las graves crisis por las que atraviesa el país, deja sentir en el ámbito local y provincial los principios ilustrados en la educación, que entre sus prioridades tiene la educación de las mujeres, dado que haría de ellas mejores madres. Su interés y su apoyo fue fundamental para enseñar a las jóvenes e implicarlas en el ámbito laboral, según se puede apreciar en la Memoria de 1873, cuando se hace referencia a La enseñanza de la mujer que se impartía en sus instalaciones. Entre las asignaturas que se ofertaban en La Enseñanza a la mujer están: Preparatoria, Gramática, Aritmética, Geología, Idioma francés, Solfeo de $1^{\circ}, 2^{\circ}$ y $3^{\circ}$ curso, Piano, Dibujo de figura, Geometría, Historia Natural y Fisiología 9 . Será en el colegio de la Sociedad Económica de Amigos del País donde Carmen Perujo iniciará sus estudios.

Es importante destacar que entre los años 1931-1938 las mujeres representaron el 8,8\% en la universidad, que en 1940 eran el 14\%, en 1970 el $31 \%$ y en 1975 ya representaban el $38 \%{ }^{10}$. El incremento es más que notable, pero es una consecuencia lógica de la alfabetización. En los años 80 llegará al 50\% en los estudios de enseñanzas artísticas ${ }^{11}$.

\footnotetext{
Richmond, 2004, 27

Capel, 1982, 362

Capel, 1982, 375

Capel, 1982, 421

Calderón, 1993

López De La Cruz, 2002

V. De Foronda, 2010
} 
La década de los años treinta en España es el período en que se presentan a un tiempo, los grandes avances para las mujeres y los mayores retrocesos en su situación jurídica y social. La llegada de la $2^{\mathrm{a}}$ República abrió la posibilidad de reformas legislativas y, aunque el ambiente no era del todo favorable, se aprovechó el momento. En contra estaban la tradición, la influencia conservadora de la Iglesia y el poco interés de los partidos progresistas, como se presenta a continuación.

En 1931, en España, La Constitución de la República reconoce el sufragio universal gracias al empeño personal de Clara Campoamor. Este mismo año en Sevilla tiene lugar la llamada Sanjurjada, liderada por el general Sanjurjo. Tendrá una trascendencia para España en aquel momento inimaginable, tal y como explica Espinosa (2005). En las elecciones legislativas del 19 de noviembre de 1933, las mujeres españolas ejercen por primera vez su derecho al voto, gracias a la defensa de Clara Campoamor. "Entramos cuando la casa se hunde" le observaba en 1931 la escocesa Chrystall MacMilan, amiga de María Lejárraga, secretaria general de la IWSA ${ }^{12}$, refiriéndose a que las mujeres habíamos logrado la ciudadanía cuando el sistema político y parlamentario entraba en crisis ${ }^{13}$. A las pioneras en el arte como Maruja Mallo, que había estudiado en Bellas Artes en la Escuela de San Fernando en Madrid, de 1922 a 1926, : se las descalifica sistemáticamente:

Garnelo, profesor de estatua, solía suspender sistemáticamente a las mujeres en el primer curso, opinaba que aquel no era lugar para ellas, decía que lo hacía por su bien, ya que de esta forma la que tenía verdadera vocación estaba llamada a perseverar, eliminando de un plumazo a las que no pensaban dedicarse a la pintura ${ }^{14}$.

En 1934, nacen Esther Ortego y Carmen Laffón de la Escosura. Esta última, ingresará en 1949 en la Escuela de Bellas Artes de Santa Isabel de Hungría, donde coincide con Teresa Duclós, nacida el mismo año, así como también Luís Gordillo, Paco Cortijo y Jaime Burguillos ${ }^{15}$. Carmen Laffón, pintora y escultora, será Premio Nacional de Artes Plásticas y Académica de la Real Academia de Bellas Artes de San Fernando. En el año anterior, 1948, Carmen Perujo ingresará en la Escuela de Santa Isabel de Hungría y sus vidas transcurrirán paralelas.

El 18 de julio de 1936 tiene lugar el alzamiento militar contra el gobierno republicano legítimamente establecido. El 30 de agosto del mismo año el $A B C$ de Sevilla publica: "se piden penas de muerte, pero la impresión es que los fusilarán a todos" 16 . Al tiempo que en Sevilla se vivía una época de fiestas sociales, bajo el férreo control del general Queipo del Llano ${ }^{17}$.

La educación, inmediatamente después de sublevación militar, pone un especial énfasis en garantizar que la escuela se encuadrara sin fisuras al servicio del nuevo estado. La represión del magisterio republicano adquiere tintes dramáticos por su repercusión social y por lo simbólico del proceso. Se crea un marco regulador específico para depurar al magisterio: Orden de 19 de agosto de 1936, BOE del 21 de ese mismo mes de agosto, para el magisterio; Decreto-Ley de 5 de diciembre de 1936

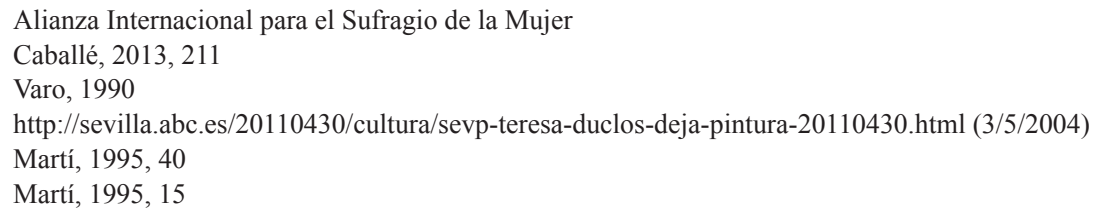


dictando reglas para la separación definitiva del servicio de toda clase de empleados, BOE de 9 de diciembre de $1936^{18}$.

Carmen Perujo, cuando habla de su infancia no habla de la Guerra, ni del hambre posterior. Solo habla del mercadillo de la calle Feria, de la pensión, de los estudios. Cuenta que al tener la salud delicada pasó grandes temporadas en el campo. La explicación de este silencio podría estar en la descripción de la situación en todo el país hecha por José Martí en La España del estraperlo:

Septiembre de 1936 se cerraba con un mal presagio para las dos Españas: la comida empezaba a escasear, sobre todo en las grandes ciudades. En la zona de la República, los campesinos escondían sus cosechas antes de que los elementos incontrolados se las requisasen". ${ }^{19}$

Las cartillas de racionamiento en Madrid dan por persona:

Medio litro de aceite, un bote de leche condensada o 100 gramos de bacalao una vez por semana. Dos huevos o cien gramos de arroz dos veces a la semana y cien gramos de legumbres, medio kilo de pan o cien gramos de carne que se pueden comprar todos los días. ${ }^{20}$

Tras el fusilamiento de José Antonio en una cárcel republicana en Alicante, Franco se hace con la dirección del partido para dar legitimidad a su golpe de estado y a su régimen. Cinco meses después de la muerte de José Antonio, el Decreto de Unificación puso a todos los partidos bajo la égida de la Falange, mientras Franco asumía su dirección. La Falange se convirtió en el partido único del Estado Español ${ }^{21}$. La Sección Femenina funcionará como difusora de los valores morales y políticos del régimen de Franco: una vuelta a la sociedad patriarcal y el restablecimiento de los roles tradicionales de ambos sexos. La Falange repararía el supuesto daño causado por la ley de divorcio reafirmando la primacía de la familia y devolviendo a las mujeres su lugar tradicional en el hogar.

En julio de 1936 la Sección Femenina tenía delegaciones en 18 provincias y sumaba 2.500 afiliadas. Al final de la guerra sumaba 580.000 mujeres según la organización. El coste de la guerra se cifra en trescientos mil millones de pesetas del año 1936 por parte de los dos ejércitos, la "inversión más estéril en la historia de España"22. El 1 de abril de 1939 el Cuartel General de Francisco Franco emite el que será último parte de guerra: "En el día de hoy, cautivo y desarmado el ejército rojo, han alcanzado las tropas nacionales sus últimos objetivos militares. La guerra ha terminado." El parte lo firma el Generalísimo. El ministro de la Gobernación firmará de inmediato la siguiente orden: "Se sustituye por Año de la Victoria el III Año Triunfal"23.

Una de las primeras medidas llevadas a cabo por el bando de los nacionalistas será la reforma del ordenamiento jurídico, imponiendo un modelo claramente conservador y paternalista que incapacitaba a la mujer para la ciudadanía y la sometía

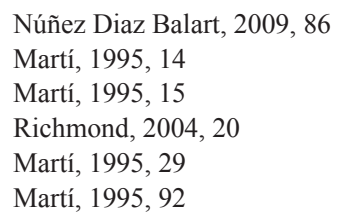


a la autoridad paterna o marital. Se releva a la mujer a la esfera de lo privado donde queda sometida a la tutela del varón. La situación de las mujeres que estaban inmersas en el mundo de la cultura, precursoras en la genealogía del pensamiento generado por mujeres en España es atroz:

Además de los asesinados, con formación de causa o sin ella, durante el proceso de depuración del magisterio español resultaron sancionados en torno a dieciséis mil maestros y maestras, alrededor del 25\% del cuerpo. Casi el 10\% fueron expulsados del ejercicio de la profesión. Francisco Morente, de quien proceden las cifras, ha llamado la atención sobre la importancia cuantitativa y cualitativa de los cargos formulados por motivos religiosos o morales, que podían agravarse en "diversa medida según la publicidad que tales actuaciones o manifestaciones hubiesen podido tener, siendo consideradas especialmente intolerables las que se hubiesen dado en el marco escolar"24.

Tras el final de la Guerra Civil, en España se instaura una metodología para el aprendizaje forzoso de los cánones ideológicos y religiosos desde el totalitarismo franquista. La instrucción académica se va a acompañar de todos los instrumentos coercitivos del Estado para lograr una imposición de la ideología nacional católica. El ciclo de cambio y borrado de la memoria no concluye hasta el inicio de los cincuenta con el final de la autarquía.

El 1 de septiembre de 1939 Alemania invade Polonia siendo considerado este momento el comienzo de la Segunda Guerra Mundial. Carmen Perujo Guerrero, tiene 9 años y asiste a la escuela primaria, al Colegio de Amigos del País. Rosario Ruiz Franco en ¿Eternas menores? Las mujeres en el franquismo destaca, y esto explica la importancia que el tema tiene para la semblanza de la biografía de Carmen Perujo, que frente a las interpretaciones que dan quienes luchan por la recuperación de la memoria histórica, están quienes consideran que el debate no es académico, dado que en los últimos años de democracia española se ha querido olvidar la dictadura, llevando a cabo un "pacto de olvido" a la hora de analizar el franquismo, constituyendo en su opinión "una tergiversación alevosa del pasado" 25 . Analiza Ruiz Franco que España:

En 1940 se enfrentó a un aislamiento internacional. El régimen desarrolló una acción destructiva del edificio construido, denostándolo de forma intensa, para después poder construir el Nuevo Estado en el solar libre y desolado. El proceso, basado en una abundante legislación, fue diseñado de forma perfecta para la persecución, para la represión, e implantó un régimen de terror que tuvo como consecuencia la extensión del miedo primero, medio importante de control social, después la resignación y finalmente la construcción de un manto de olvido en amplias capas de la sociedad española. Esa acción, con duración similar a la vida del franquismo, fue especialmente intensa en los años cuarenta, verdadera década ominosa de nuestra historia, ayudada por la impunidad internacional que brindó a Franco la Segunda guerra Mundial, el apoyo de la iglesia católica con su amplia red de sociabilidad y la extensión del estado de guerra hasta $1948 .^{26}$

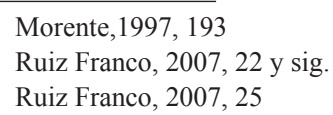


En 1940 Carmen Perujo tiene diez años. Será con catorce que comience su formación como artista. De modo que sus años de primera formación, también los posteriores, se verán mediatizados por el cambio histórico de fundamental trascendencia que está teniendo lugar en el país. Son los años que José Martí narra en Los años del estraperlo: "A principios de los años cuarenta una familia obrera compuesta por cuatro personas que se alimentase a los precios de tasa necesitaba diariamente 9,56 pesetas" 27 . La familia de Carmen consta de seis miembros: su padre y su madre, sus hermanas Ángeles, Pastora y Rosa, y ella. Además, debemos tener en consideración que sus progenitores regentaban una pensión. Por tanto, podemos arriesgar a imaginar lo importante que era el estraperlo en sus vidas.

No se puede comprender la creación de la obra de Carmen Perujo, la elección de su tema de desarrollo vital, sin comprender el borrado de memoria y el secuestro de identidad que se estaba llevando a cabo sobre la memoria colectiva de las mujeres españolas durante los años cuarenta. Hay que comprender que una herramienta fundamental de este cambio, además de los cambios legislativos, fue la tergiversación de la educación y la política cultural.

En este momento de ausencia de pensamiento crítico, es necesario nombrar a D'Ors y tanto su Academia Breve de Crítica de Arte como sus Salones de los Once, que tendrán continuidad durante toda la década de los cuarenta, siendo a medias asambleas de relaciones sociales y a medias manifestaciones artístico intelectuales. Gracias a estas reuniones se publica la revista Santo y Seña cuyo último director es Castro Arines. Posteriormente Santo y Seña se reactivará en Arte y Letras $^{28}$. Esta academia Breve quería ser una iniciativa moderada de enlazar con el arte moderno y ponerlo en valor para salir del ambiente vacuo de la postguerra. En estos Salones de los Once solo estaba presente una mujer: La Condesa de Campoalange, María Laffitte. Se realizaba una celebración anual del Salón donde cada miembro de la Academia debía presentar a un artista. La Academia Breve y sus exposiciones tuvieron cierta "oficialidad" y respeto, lo que redundó en beneficio de esos jóvenes artistas. En este ambiente, María Laffitte rescatará para la memoria de las mujeres la figura de María Blanchard.

A partir de 1941 se comienza a publicar la revista de la Sección Femenina Consigna que "daba a sus lectoras pautas educativas siempre presididas por la religión. Sugería como materia de enseñanza lo que el profesor Tuñón de Lara llamó alguna vez «interpretación oficial de la Historia»" 29 ofreciendo una visión de la realidad en la que Francisco Franco aparecía como la clave de España conformando una historia de las mujeres desde las grandes consignas de la Sección Femenina. Se terminaría creando así una cultura femenina y utilitarista que conjuga ese sentido «misional», social y patriótico, con el conocimiento sobre el deber conyugal. El régimen reinstaura como función de las mujeres en la sociedad, la de ser esposa y madre. El valor social femenino va unido a lo íntimo de las familias, y no a lo público y externo, monopolio del hombre. En el nuevo ordenamiento jurídico, las mujeres son seres obligados a una función social específica, basada en su capacidad reproductora y educacional.

La formación de las mujeres más jóvenes se vería complementada con la obligatoria adhesión de todos los escolares al Frente de Juventudes y la asistencia a campamen-

\footnotetext{
Martí, 1995, 75

VV. AA., Madrid: el arte de los 60, 1990, 51

Gallego Méndez, 1983, 156
} 
tos, albergues y, sobre todo, a las Tardes de Enseñanza, donde las niñas completaban su visión genealógica y cosmológica de la Historia de la Falange y de España, como una «unidad de destino en lo universal». Acción Católica, el Opus Dei, la OJE, a través de sus Montañeras de Santa María y otras organizaciones afines al Régimen, serán las depositarias del tiempo de ocio de las mujeres. Se conforma así un ideal histórico-católico de feminidad, coincidente con el modelo impuesto por la Iglesia.

En resumen, con la llegada del Franquismo, las misiones pedagógicas, el servicio de bibliotecas y otro tipo de actuaciones que sustentaban el sistema educativo, social y democrático, de la República se terminan. Pero no solo se dan por finalizados, sino que se borran de la memoria colectiva y no serán recuperados hasta el final de los años 90 del siglo XX. Se defiende la tradición y la religiosidad y se deja en manos de la Iglesia Católica gran parte de la labor educativa. Se crea una conciencia nacional única a través de la uniformidad lingüística y cultural.

Es posible que a la provincia de Sevilla donde Carmen Perujo está fraguando su vocación artística, pudieran llegar ecos de la resistencia republicana. Es fácil que, para una niña de la posguerra de catorce años, hechos como la presentación de María Blanchard por parte de Laffitte en el Salón de los Once o el regreso de Menchu Gal a Madrid, no tuvieran la importancia que podía tener la supervivencia en la cotidianeidad y, sin embargo, a nivel colectivo, se podrían considerar ecos de la República.

El año de 1944, cuando Carmen termina sus estudios en el colegio, sabemos de las necesidades familiares, de todo tipo, y las manos que hacen falta para atender la pensión de la Calle del Aire, arrimando el hombro aún a costa de interrumpir las clases en el Colegio de Amigos del País ${ }^{30}$. "Con lo chica que es y lo espabilada que está, yo de ti la dejaría ir a la escuela un año más Esteban” cuenta Carmen que le recomendaba un albañil amigo a su padre. Y así, logra Carmen ir un año más a la escuela, mientras sus hermanas ayudaban a su padre y su madre en la pensión, su encargo era comprar todos los días el pan, ayudaba un poco y cuando terminaba iba en el tranvía hasta la escuela a todo correr para no llegar tarde.

\section{Juventud y estudios}

En septiembre 1944, recordamos que Ana de la Paz Perujo ${ }^{31}$, nombre con el que Carmen Perujo consta en su primera documentación académica, tiene 14 años, e ingresa en la Escuela de Artes y Oficios, superando con calificación de sobresaliente el primer curso, y sobresaliente, premio de asistencia y primer premio en el segundo curso. Todo en el año escolar de 1944 - 45, según consta en su cartilla de calificaciones.

En esos años la Escuela de Artes y Oficios estaba instalada en el Pabellón Mudéjar de la Plaza de América, donde permaneció Carmen cuatro años de iniciación en las artes plásticas, con gran aprovechamiento bajo la tutela del profesor Rodríguez Jaldón, del que guarda un grato recuerdo. Sus calificaciones son extraordinarias: en el curso 1945 - 46 obtiene sobresaliente y segundo premio en el tercer curso y notable y premio de asistencia en Conpt $^{\circ}$ del Arte y en Historia de las Artes Decorativas.

\footnotetext{
30 No existe mucha información de Colegio de Carmen Perujo y todo es anterior a 1900. Por ejemplo, la siguiente tesis doctoral que habla de los centros educativos derivados de las Sociedades de Amigos del País: http://fondosdigitales.us.es/media/thesis/636/15_CAPITULO_6.pdf

31 Documentación del archivo personal de Carmen Perujo Guerrero
} 
En el siguiente curso de 1946 - 47 también obtiene sobresaliente, primer premio y premio de asistencia en el $4^{\circ}$ curso de dibujo artístico en la Escuela de Artes y Oficios de Sevilla ${ }^{32}$.

En estos años Carmen acompaña a su padre al mercado de la calle Feria. Allí le veía elegir los cuadros con que adornar la pensión en el Mercado de los Jueves ${ }^{33}$, el mercadillo al aire libre más antiguo de Sevilla. Imaginamos la escuela que para Carmen supone el acompañar a su padre todas las semanas al mercado en estos tiempos de postguerra, en los años del estraperlo.

En 1946 la Asamblea General de la ONU recomienda en una resolución que se excluya al Gobierno español de Franco como miembro de los organismos internacionales establecidos por las Naciones Unidas o que tengan nexos con ellas. Recomienda a todos los miembros de las Naciones Unidas que retiren inmediatamente a sus embajadores y ministros plenipotenciarios acreditados en Madrid. Casi todos los países miembros, a excepción de Portugal y Argentina, siguieron las recomendaciones de la ONU.

En estos mismos años se llevaba a cabo la evolución, desarrollo y mutuo conocimiento de los artistas de Ariel y Els Blaus, en Cataluña. Y la creación en 1946 del grupo Dau al Set. En Andalucía, la región de nuestra artista, y más concretamente en Almería, se crea en la década de los cuarenta el Grupo Indaliano. Las bases del grupo las pone en 1946 su fundador Jesús de Perceval. Busca un resurgir clasicista y en él se integran Capuleto, Castellón, Cantón Checa, Cañadas, Gómez Abad, Enrique Suárez, López Díaz, Miguel Rueda, Garzolini, Tola, Anchoniz, Fernández Piár, Alcaraz y Cuadrado. Grupo que en la I Bienal Hispanoamericana de 1951 será el que más se mencione, tanto para la selección como para las polémicas. Los ecos de esta Bienal Hispanoamericana tienen que llegar a la joven recién egresada de la Escuela de Bellas Artes, Carmen Perujo, protagonista del presente artículo y, podemos suponer, que debieron de darle alas, ya que un acontecimiento solemne que tenga que ver con la profesión elegida por una joven de veinte años es vivido con avidez y expectativas.

El aislacionismo incrementa en España el impacto negativo de la posguerra y ahonda la pobreza, de la que el país no se recupera hasta los años 50, cuando, con motivo de la Guerra Fría, las potencias occidentales necesiten las bases españolas y el régimen franquista se convierta en un "mal menor" desde el punto de vista de Estados Unidos y sus aliados. En los años 50 Carmen Perujo ya tiene veinte años y estos años de guerra y posguerra serán determinantes para su "hechura" artística.

Es un tiempo que socialmente difunden insidias sobre la moralidad y sobre ella se asientan los delitos, pasando el pecado a ser delito, se logra que las mujeres callen y un absoluto silencio para sí y para los hijos e hijas, se difunde la vergüenza que provoca el silencio, el "cállate, porque tú no puedes hablar", el "de esas cosas no se habla fuera de casa", el "eso no se dice". La pérdida de la identidad propia y familiar parte de la ocultación de toda una procedencia y una actuación que se había convertido en testimonio de cargo sobre las mujeres y sus entornos. La dignidad de las mujeres se une a la moral privada y pública ${ }^{34}$, quedando así las prestaciones

Documentación del archivo personal de Carmen Perujo Guerrero

$33 \mathrm{http}: / /$ hemeroteca.sevilla.abc.es/nav/Navigate.exe/hemeroteca/sevilla/abc.sevilla/1983/02/24/035.html (20/9/2012)

34 Nuñez Diaz-Balart, 2009, 134 
por parte de las mujeres al servicio de los vencedores y varíando dependiendo del poder más próximo: unas veces se trata de barrer la iglesia, la casa del señorito o el cuartel local de la Falange. En los pueblos, se da el caso de que las viudas tengan que tramitar la pensión, que es lo que les permite la supervivencia, a través del sacerdote, lo que conlleva la obligatoriedad de cumplir con la misa dominical y otras similares.

La doctrina parte de una supuesta enfermedad moral y genética que padecen las mujeres "rojas", según Antonio Vallejo Nájera, el jefe de los Servicios Psiquiátricos del Ejército franquista, profesor de la UCM y miembro de la Real Academia de la Historia, con todos los honores ${ }^{35}$.

Los términos de maternidad, infancia protegida, hogar y familia, están constantemente en boca del régimen, mientras que la realidad de las mujeres, jaleada por la propaganda oficial, afronta una situación de subsistencia y adaptación en la que se prescinde de todo asomo de individualidad.

En el caso de las mujeres artistas esta circunstancia está siempre presente, pero nunca se menciona. Cuenta Amalia Avia, cuyo marido era Lucio Muñoz, cuando vende su primer cuadro la insatisfacción que le provoca el venderlo a un amigo:

Lo que yo quería era vender un cuadro de verdad, a un desconocido, sobre todo porque esto me afianzaría ante mi familia; por lo demás, estaba tan segura de que no iba a ganar jamás dinero con la pintura, que el hecho no me afectó demasiado ${ }^{36}$.

Es en este contexto que tienen lugar los hechos que Carmen narra sobre la pequeña pensión que su familia tenía: contaba con 26 habitaciones y su padre le dejaba una habitación para pintar. Una habitación y las sábanas: las sábanas para pintar y la "habitación propia" para crear. Esto fue determinante para su elección de vida ya que le facilitó el espacio y el material. En ocasiones no se tiene en consideración lo importante que es para las mujeres artistas en ciernes tener disponibilidad de espacio y materiales para llevar a cabo la primera obra creativa.

En estos años duros de la postguerra y la resignificación del imaginario colectivo, Carmen Perujo lleva a cabo sus estudios de Bellas Artes en lo que a día de hoy es la Facultad de Bellas Artes de la Universidad de Sevilla. Años hechos de represión, silencio y borrado de la memoria de la historia de las mujeres. Quizás la principal consecuencia de este borrado de memoria fue la asunción absoluta por parte de la mayoría de las mujeres españolas de que su verdadera carrera sería ser madre de familia.

El año que Carmen Jiménez, escultora sevillana, se casa con el escultor Antonio Cano Correa, 1947, podemos tener la sospecha de que a Perujo le llegara algún eco de esta boda y de la existencia de otra escultora, aunque ella no pueda confirmármelo. Es el momento en que Carmen Perujo finaliza sus estudios con sobresaliente y primer premio en Dibujo de lo antiguo, sobresaliente en Anatomía pictórica, sobresaliente en $1^{\circ}$ de modelado y vaciado, sobresaliente y segundo premio en colorido y composición, sobresaliente y primer premio en $2^{\circ}$ de modelado y vaciado (a 30 de

Nuñez Diaz-Balart, 2009, 136

36 Avia, 2004, 236 
junio de 1948). También podemos constatar que en el año 1948 Delhi Tejero gana el concurso para el mural del Ayuntamiento de Toro que titula Amanecer jurídico del municipio zamorano que termina de ejecutar en 1950 y a cuyos materiales incorpora el oro.

Perujo cuenta que en esta época los síntomas de rabia de un gato que muerde a su padre aconsejan un tratamiento antirrábico, al cual le acompaña su hija pequeña. Es en la Puerta del Osario donde van a que su padre reciba la inyección, este queda muy cerca del edificio de la Escuela de Bellas Artes de Santa Isabel de Hungría, y Carmen le pide a su padre que la deje asistir a sus clases y este accede. Solicita una beca y se le conceden 300 pesetas mensuales. Llega a un acuerdo con sus hermanas, que se ocuparán del trabajo que le corresponde en el negocio familiar y entrega toda su energía a los estudios de Bellas Artes, que tienen lugar en la que fuera residencia del pintor Gonzalo Bilbao y cuyo año preparatorio le llevará el curso 48- 49. Apareciendo en su documentación que:

Doña Carmen Perujo Guerrero, es alumno oficial de esta escuela en el presente año, matriculado en el curso preparatorio libre, Sevilla, el 6 de noviembre de 1948. Visado para este año, consta matriculado en el curso Ingreso preparatorio. Sevilla, 10 de abril de 1949. Visado para este año, consta matriculado en el curso $1^{\circ} \mathrm{de}$ Pintura, Sevilla, 9 de septiembre de $1949^{37}$.

Como vemos, inicia la especialidad de pintura. También encuentra a uno de los profesores con los que conserva reconocimiento y amistad: Miguel Pérez Aguilera.

En el año 1948 María Lafitte, Condesa de Campoalange publica con la Revista de Occidente, "La guerra secreta de los sexos" contando con la oposición de Eugenio D'Ors quien, además de recomendarle a Otto Wieninger, le dedica cinco duras glosas con el título "La secreta paz de los sexos".

Este año Maruja Mallo y Marisa Roesset participan en la exposición "Arte Contemporáneo Español" en el Museo de Bellas Artes de Buenos Aires ${ }^{38}$. Imposible saber si los ecos de estas actividades pudieron llegar a Sevilla.

En 1949 Carmen Jiménez obtuvo el gran premio del Círculo de Bellas Artes de Madrid, premio en el que competía con Juan de Ávalos y Juan Cristóbal, con la obra llamada Eva, una pieza de $1.80 \mathrm{~cm}$ tallada en madera representando una mujer con la manzana en la mano derecha ${ }^{39}$. Es una figura robusta, pletórica de vitalidad y con la cabeza erguida, de tradición modernista que podemos contemplar a día de hoy presidiendo la cafetería del Círculo de Bellas Artes.

Las palabras de Natalia Seseña ${ }^{40}$ que se licencia en Historia del Arte por estos años, explican muy bien el tipo de descalificación que podía recibir una estudiante por aquellos entonces:

A pesar de la gran maestría -en general- de los profesores que tuve en la Facultad de Filosofía y Letras, ya que muchos se habían formado con la Junta para Ampliación de Estudios, tenían hacía las chicas una actitud de desconfianza ante

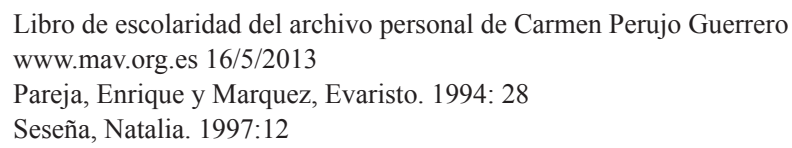


su vocación profesional. Recuerdo que cuando se aproximaba el fin de la carrera pensé en varios temas para mi tesis doctoral. Siendo habitual de la biblioteca del Ateneo, había empezado a leer algunos libros "prohibidos" sobre la influencia de la psicología en la historia. Se me ocurrió que un asunto interesante sería estudiar los rasgos esquizofrénicos de los reyes y príncipes de la Casa de los Habsburgo. Equivocada o no se lo propuse a don Cayetano Alcázar quien me miró de arriba abajo y de forma desparpajada me dijo: "Mire señorita, yo creo que usted es muy joven y que mejor será que no piense en la tesis doctoral y que se case". Mi tesis -mucho más humilde- se retrasó años. Mientras, fui becaria en EEUU, y, efectivamente me casé".

Carmen, en la narración de vida que constatan las entrevistas, no se detiene en el relato de su infancia o en los relatos de sus experiencias en la carrera, salta rápidamente a su vida expositiva y profesional, no quiere personalizar ni individualizar e intenta ser aséptica en su vida personal. En las entrevistas se detecta constantemente la necesidad de "discreción".

La política cultural del franquismo, prácticamente inexistente, ante la ausencia de vanguardia, toda en el exilio debido a la Guerra, enfoca el favor oficial hacia el arte academicista que es lo que queda a flote en estos años. Las Exposiciones Nacionales de Bellas Artes, fundadas por Isabel II en 1854, son el canal oficial que acapara las exigencias académicas. También están los "Salones de Otoño", que son "semioficiales”, dependientes del Salón de los Once de Eugenio D`Ors, y que durante los años cuarenta además de bajar su calidad son un reducto de academicismo que imponen un "desanimante y cerrado tradicionalismo artístico" 41.

En estos años, la década de los 50, quedan unas cuantas galerías de arte que dieron alguna oportunidad de desarrollo a las vanguardias. En Madrid serán Biosca, Clan, Abril, Estilo, Xagra, Turner y Buchholz, esta última dará nombre al grupo de artistas que con ella trabajaban. Estas galerías, al igual que las de Barcelona, serán decisivas en la selección de obras para la I Bienal Internacional Hispanoamericana que marcará un antes y un después en la historia del arte contemporáneo del siglo XX español. En 1948 Mathías Goeritz "descubre” las cuevas de Altamira y junto a Ricardo Gullón, Pablo Beltrán de Heredia y Ángel Ferrant, fundan la Escuela de Altamira en Santillana del Mar y aúnan esfuerzos para enlazar con la vanguardia rota por la Guerra Civil. Crean en el año 1949 la revista Bisonte que publica monografías de Lloréns Artigas, Ferrán y otros, y alguna exposición. También crea la Primera Semana Internacional de Arte Contemporáneo en septiembre de 1949 y una segunda en septiembre de 1950 acompañándola de una exposición de composiciones abstractas de Carla Prina (1911-2008) en la Sala Proel ${ }^{42}$. En 1949 Eusebio Sempere expone sus formas abstractas en la valenciana Galería Mateu que tendrá un gran éxito mediático generando gran polémica.

En septiembre de 1950, Carmen Perujo consta matriculada de manera oficial en Artes y Oficios. En el curso 1951 -52 está matriculada en $3^{\circ}$ de Pintura y en el curso 1952- 53 en el curso de Profesorado. Del 1 de octubre de 1951 es su pase oficial al interior de los palacios y jardines de los Reales Alcázares de Sevilla, con el nombre

\footnotetext{
Cabañas Bravo.1996, 59

42 Cabañas Bravo.1996, 70.
} 
de Carmen Perujo Guerrero ${ }^{43}$, que le daba derecho a pintar del natural en un espacio exclusivo. En estos dos años, nuestra artista, logra un premio del Estado, en metálico, en los cursos 50-51, y otro igual en el 51-52 como consta en el Certificado, con fecha de 1961, que hace la Universidad de Sevilla.

En la Universidad Española donde Carmen Perujo estudia, impera el Sindicato Español Universitario (SEU), constituido en 1933 para oponerse, incluso de modo violento, a la FUE (Federación Universitaria Escolar) y nombrado, por decreto de Franco, el único sindicato estudiantil en 1939: cualquier persona que quiera obtener un título superior, tiene la obligación de afiliarse a él. Así es que lo hace Carmen Perujo cuyo carné de afiliada al SEU, esta vez con el nombre de Ana de la Paz, y con fecha de admisión del 19 de octubre de 1952, al mismo tiempo que está matriculada en el curso de Profesorado de Dibujo.

Los "pequeños libritos" publicados por Acción Católica proliferan entre el estudiantado femenino haciendo proselitismo de cómo se debe vivir con párrafos como el siguiente:

Joven que en el estudio aprendes sacrificio y vencimiento propio, al tiempo que la conciencia va abriendo surco en ti. ¿Verdad que tu ilusión va lejos y se extiende y domina a los deseos materiales de lograr una posición?

Me acerco a ti para, de igual a igual, hablarte con a dulzura del Amor y con la fuerza de la Verdad. Quiero unir mi vida joven a la tuya, ser tu hermana y hacer contigo la jornada.

Has llegado a la Universidad. Pasaron ya los días de colegio y tal vez está lejos la amorosa mirada de tu madre, en vigilia continua por mantenerte sencilla y virtuosa y hacerte crecer en bondad, a medida que lo hacías en edad y cultura. Si es así, sientes la ausencia del hogar amable, donde las horas tienen el sello del trabajo y la paz de las conciencias que cumplen su deber.

Estos libritos están dirigidos a una población exclusivamente femenina, (los hombres tienen su propia "literatura") son ellas las que deben ser sencillas, bondadosas, virtuosas... el Régimen necesita de mujeres sumisas para poder establecer su filosofía nacional católica.

El panorama que dibuja las relaciones entre los artistas contemporáneos (sobre todo los y las artistas de la transición) se aboceta estos años en la Escuela de Bellas Artes de San Fernando de Madrid, al igual que hemos visto que pasaba en la Escuela de Santa Isabel de Hungría de Sevilla. Muchos de los artistas contemporáneos a Carmen Perujo estudian durante estos años en la Escuela de Bellas Artes de San Fernando.

A partir de 1952 se encuentran en torno al arte y la literatura una generación de artistas de los cuales, algunos, acabaran casándose entre sí, como son los casos de Antonio López y María Moreno, Lucio Muñoz y Amalia Avia, Francisco López e Isabel Quintanilla y Julio López y Esperanza Parada. Algunos formarán parte de la Escuela del Realismo Madrileño. Carmen Perujo, sevillana, se casa con Arcadio Blasco, valenciano, y trabajan en sus primeros años mano a mano con parte de esta "generación".

Carmen Perujo tiene 19 años y está en Sevilla finalizando sus estudios en el tiempo en que Beauvoir publica, en la Francia de la posguerra, El Segundo Sexo. Beau-

43 Hemos de recordar que en otros documentos consta como Ana de la Paz 
voir aborda la situación de las mujeres desde todos los puntos de vista y desde todos los espacios, desde lo público y desde lo privado. Analiza las contradicciones de la mujer, como producto de ser "la otra", lo que Beauvoir denomina otredad o alteridad, incluye su obra personal en ello: una filósofa existencialista que, a los 40 años, escribe una obra que ha abierto los ojos a millones de personas de varias generaciones. A la sombra del pensamiento de Beauvoir se puede parafrasear: No se nace artista, se llega a artista.

No se nace mujer: se llega a serlo. Ningún destino biológico, psíquico, económico, define la imagen que reviste en el seno de la sociedad la hembra humana; el conjunto de la civilización elabora este producto intermedio entre el macho y el castrado que se suele calificar de femenino. Solo la mediación ajena puede convertir un individuo en alteridad" 44 .

Este año se asiste a la creación de colectivos que marcarán la segunda parte del arte español del siglo XX. Se crea en Las Palmas de Gran Canaria el grupo LADAC (los arqueros del arte contemporáneo) compuesto entre otros por Millares, Fleitas, Monzón y Elvira Escobio ${ }^{45}$. En julio de 1951 todo el grupo expondrá en la Galería Syra de Barcelona ${ }^{46}$ y en el 55 llegan a Madrid ${ }^{47}$.

En 1950 se convocan dos concursos públicos que tendrán mucha importancia en el panorama artístico español y que generarán imaginario colectivo: la construcción de la Basílica de la Santa Cruz del Valle de los Caídos, en Cuelgamuros ${ }^{48}$ y el concurso para la reconstrucción de la Basílica de Aranzazu. Desde un primer momento van a plantear dos modos de hacer arte, siendo derrotistas podríamos decir que Aranzazu representa la España que quiere ser y el Valle de los Caidos la España que es.

Otro evento artístico en 1951, año en que Carmen Perujo termina sus estudios, es la I Bienal Hispanoamericana de Arte. El proyecto está en marcha antes del cambio ministerial que lleva a Ruiz Jiménez a la cartera de Educación, pero se retoma para comenzar una política cultural que proyecte una imagen de modernidad y benevolencia que no se corresponde con la realidad. La convocatoria de la I Bienal Hispanoamericana de Arte y las sucesivas ediciones ofrecen la posibilidad de romper el monopolio ejercido por el academicismo. Tras la I Bienal se abre un clima que permite la recuperación de las nuevas tendencias del arte joven y su presencia en la cotización internacional

Carmen Perujo tiene a gala que sus padres decidieran invertir en su educación y que su formación fuera la mejor posible: en las entrevistas concedidas a la autora de este artículo lo manifiesta de manera reiterada. Tuvo buenos profesores y le llevaron al colegio de la Sociedad Económica de Amigos del País: uno de los colegios más abiertos de Sevilla, donde en los primeros cursos los niños y las niñas aprendían juntos, en el mismo aula, con un profesorado progresista. Perujo también tiene a gala

$44 \quad$ Beauvoir, 2010, 371

45 E. Escobio se casaría más tarde con Manolo Millares y solo la he encontrado mencionada, además de la cita de Cabañas Bravo, en el documental que sobre Millares hace su hijo en el año 2005, donde deja dicho "Soy la viuda de Manolo Millares, pero soy Elvireta Escobio antes que nada" y donde dicen que ella estaba "enormemente dotada para el arte" Millares Alonso, Juan. Cuadernos de contabilidad. 2005 y en la obra de Cabañas Bravo

46 Castro, 1990: 48

47 Millares, 2005

48 Bazán De Huerta, 1996 
que estaba "retirado de casa" y que ella "iba sola". Por el barrio de Santa Cruz no había colegios para niños. Ello lleva a que su trato con los chicos fuera desinhibido $\mathrm{y}$ sin miedos.

La formación de Carmen Perujo finaliza el mismo año que la autarquía. En 1951 Estados Unidos admite de muy mala gana la decisión de Franco de enviar como embajador a Washington al vasco José Félix de Lequerica, al que Truman no dio el plácet en $1945^{49}$. El 26 de septiembre de 1953 se firman los convenios hispano americanos conocidos como el Pacto de Madrid. A través de estos acuerdos España recibe un prestamo por parte de los Estados Unidos y se establecen las Bases de utilización conjunta en territorio español ${ }^{50}$.

El Régimen empieza a ser cuestionado en las calles. El primer gran desafío público es la huelga de tranvías en Barcelona, del 1 al 8 de marzo de 1951. Durante una semana la población se niega a subir a los tranvías en señal de protesta por el aumento de las tarifas. Esta huelga deja varios detenidos y el inicio de una serie de huelgas laborales en cadena, que suponen el cese del Gobernador Civil, del delegado provincial de Sindicatos y del jefe superior de policía, y el embrión de una crisis: unos meses después, en julio de 1951, el gobierno sufre el cambio más profundo desde final de la guerra: once nuevos ministros entran en el gabinete ${ }^{51}$.

El nuevo gobierno marca un giro en política, ya que es el gabinete el que emprende la liberalización económica. En este nuevo gobierno se nombra como subsecretario de la Presidencia a Luís Carrero Blanco y, en la cartera de Información y Turismo, a Gabriel Arias Salgado. Esta composición se mantiene hasta 1957, salvo dos cambios en 1956. Lo que pasa a la historia como "huelga de los tranvías" tiene como trasfondo el malestar social por las condiciones de vida marcadas por el racionamiento, el estraperlo y las continuas restricciones en los suministros de luz, de gas y de agua.

\section{Conclusiones}

La formación recibida en una escuela laica como Los amigos del País, y la posterior en Artes y Oficios y en Bellas Artes nos presenta una artista sobradamente cualificada para ejercer el oficio de la pintura,

El hecho de que el apoyo familiar sea algo constatable, dado que la legislación ponía en manos dal pater familias la capacidad para permitir o impedir este tipo de estudios que socialmente no tenía gran aceptación y que el Régimen no facilitaba, pone de relieve la importancia de la colaboración familiar en estos años difíciles para el desarrollo intelectual de las mujeres.

Ante la escasa vida cultural, el insistir en querer educarse y progresar artísticamente hasta llegar a tener un imaginario propio, deja patente una firme vocación plástica.

Dejamos constancia de la importancia de recuperar la obra de Carmen Perujo ya que su trabajo con el barro, y la visibilidad que este tuvo en los años de la transición, motivó que muchas mujeres buscaran en las Bellas Artes su realización personal.

\footnotetext{
Martinez Lillo, 1996

Piñeiro Álvarez, 2006

Martí Gómez, J. 1995, 198
} 
No se puede llegar a comprender como es posible que realice un corpus de obra tan abundante en los años 80 sin conocer que allá por los años 50, cuando este país estaba todavía comenzando a salir de la autarquía, había una muchacha sevillana finalizando sus estudios de Bellas Artes que soñaba con ser artista.

Dejando los años de matrimonio, crianza y éxito profesional de Carmen Perujo para un segundo artículo, se concluye con la observación sobre los años de formación de esta artista, quien con un excelente expediente académico, vive una grave falta de reconocimiento histórico. Mientras este reconocimiento en la historia y en los libros de texto no tenga lugar, seguiremos repitiendo una y otra vez, al estilo de la Condesa de Campoalange, que sobre aquella que nosotras estamos escribiendo es "la primera".

\section{Referencias Bibliográficas}

Avia Peña, Amalia (2004). De puertas adentro: memorias. Madrid: Ed. Taurus Alfaguara.

Bazán de Huerta, Moisés (1996). Juan De Ávalos. Su verdad creativa. Badajoz. Edita: Caja de Badajoz

Beauvoir, Simone (1981). El segundo sexo. 2 tomos. Buenos Aires: Ediciones Siglo XX.

Caballé, Anna (2014). El feminismo en España. La lenta conquista de un derecho. Madrid: Ediciones Cátedra.

Cabañas Bravo, Miguel (1996). La política artística del franquismo. Madrid: Consejo Superior de Investigaciones Científicas.

Calderón España, María Consolación (1993). La Real Sociedad Económica Sevillana de Amigos del País: su proyección educativa (1775-1900) Sevilla: Secretariado de Publicaciones de la Universidad de Sevilla.

Campo Alange María (1990). Mi niñez y su mundo. Madrid: Editorial Castalia. Biblioteca de escritoras. Instituto de la Mujer.

Capdevila-Argüelles, Nuria (2013). Artistas y precursoras. Un siglo de autoras Roësset (1882 -1995). Madrid: Editorial Horas y Horas.

Capel Martínez, Rosa Ma (1986). El trabajo y la educación de la mujer en España (19001930). Madrid: Ministerio de Cultura. Estudios sobre la mujer.

Castro, Fernando (1990). El surrealismo a la sombra del Teide en El surrealismo entre el Viejo y el Nuevo Mundo. Madrid: Mapfre Editorial.

Espinosa, Francisco (2005). La Justicia de Queipo. Barcelona: Ed. Crítica.

Freeland, Cynthia (2010). Pero ¿esto es arte? Madrid: Ed. Cuadernos Arte Cátedra.

Gallego Méndez, María Teresa (1983). Mujer, Falange y Franquismo. Madrid: Taurus.

Laffitte y Pérez Del Pulgar, María (1983). Mi atardecer entre dos mundos: recuerdos y cavilaciones. Madrid: Editorial Planeta.

López De La Cruz, Laura (2002). La presencia de la mujer en la Universidad Española. Sevilla: Edita Universidad Pablo de Olavide.

Martí Gómez, José (1995). La España del estraperlo (1936-1952). Barcelona: Editorial Planeta.

Millares Alonso, Juan (2005). Cuadernos de contabilidad de Manuel Millares. Madrid: Productora Raccord en Escena

Morente Valero, Francisco (1997). La escuela y el Estado Nuevo. La depuración del Magisterio Nacional (1936 - 1943). Valladolid: Ámbito Editorial.

- (2001). La muerte de una ilusión: El Magisterio español en la Guerra Civil y el primer Franquismo. Revista Historia y comunicación Social, 6, 187-201. 
Nuñez Diaz Balart, Mirta et al (2009). La gran represión. Los años de plomo de la posguerra (1939 - 1928). Barcelona: Flor de viento ediciones.

Pareja, Enrique y Márquez, Evaristo (1994). Carmen Jiménez. Sevilla: Editorial Gerver, S.A. Richmond, Kathleen (2004). Las mujeres en el fascismo español. La sección femenina de la falange, 1934 - 1959. Madrid: Alianza Editorial.

Ruiz Franco, Rosario (2007). ¿Eternas menores? Las mujeres en el franquismo. Madrid: Biblioteca nueva.

- (2003). La situación legal: discriminación y reforma. En Nielfa Cristóbal, Gloria. Mujeres y hombres en la España franquista: sociedad, economía, política, cultura. Madrid: Editorial Complutense.

Seseña, Natalia (1997). Cacharrería popular. La alfarería de basto en España. Madrid: Alianza Editorial.

V. De Foronda, Pilar (2010). Análisis comparativo de licenciados/as con los académicos/as en las Reales Academias españolas que conforman el Instituto Español. Madrid: Revista del Instituto de investigaciones feministas.

Varo, Beatriz (1990). Remedios Varo: En el centro del microcosmos. Madrid: Editorial Fondo de cultura económica de España

VV. AA. Cajide, Isabel (1990). Madrid: el arte de los 60. Revista Artes. Madrid: Ed. CSIC. www.mav.org.es

http://www.fernandezdelamo.com/pueblos

http://hemeroteca.sevilla.abc.es/nav/Navigate.exe/hemeroteca/sevilla/abc.sevilla/1983/02/24/035.html

http://sevilla.abc.es/20110430/cultura/sevp-teresa-duclos-deja-pintura-20110430.html 